\title{
Improved understanding, detection, and management of neuropsychiatric complications: essential components to the optimal treatment of Parkinson's disease
}

It has been well established that Parkinson's disease (PD) is not just a movement disorder (Weintraub and Burn, 2011). Fortunately, the past two decades have seen increased attention to the neuropsychiatric aspects of the disease process. Neuropsychiatric symptoms (e.g., mood, sleep, psychosis, and impulse control) are experienced by the overwhelming majority of people living with PD (PWP) and have a detrimental impact on physical and cognitive decline (Pontone et al., 2016), quality of life (van Uem et al., 2016), and caregiving relationships (Santos-García, 2015). As a field, all multidisciplinary providers involved in the care of PWP, inclusive of movement disorder specialists, geriatric psychiatrists, clinical psychologists, social workers, and other allied healthcare providers, must work to improve the recognition and treatment of key non-motor symptoms, such as depression and anxiety, as part of the standard of care (Cohen et al., 2016). Improved detection and management is critical, as the failure to appropriately treat psychiatric complications negatively impacts the overall course of the illness, functional aspects of daily life, and the PWP ability to fully engage in their own self-care (Pontone et al., 2016).

One challenge to the appropriate detection and treatment of psychiatric symptoms in the context of PD is that these symptoms may present in unique and nuanced ways in PD compared to older adults without PD and/or with other medical comorbidities (Sauerbier and Chaudhuri, 2014). Moreover, the psychological and physical challenges of living with PD may interact with each other in ways that remain poorly described. Thus, the improved understanding of the phenomenology and clinical correlates (cognitive, behavioral, and biological) of depression and anxiety in PD may shed light on new and innovative assessment and treatment paradigms. The articles presented in this issue advance our understanding of the neuropsychiatric complexities inherent in PD from several distinct perspectives.

\section{Mild depression in PD linked to key brain changes}

We begin with a neuroimaging study by Ansari and colleagues that utilized diffusion MRI connectometry to compare differences in white matter connectivity across brain regions between depressed and non-depressed individuals with early stage PD. This study extends prior imaging research in depression in PD (dPD) by examining network connections across the whole brain versus those found only in a specific region of interest, in order to more comprehensively describe neurobiological correlates of dPD (Wen et al., 2016). Key findings revealed decreased connectivity (i.e., decreased white matter or axons) in the uncinate fasciculi, which connects the structures like the hippocampus and amygdala to the prefrontal cortex, and notably, the orbitofrontal cortex (OFC). Decreased connectivity to the OFC has been previously observed in major depression (Frodl et al., 2010) and may be associated with decreased cortical regulation of limbic activity, leading to difficulties processing negative emotions and regulating self-schemas. The second key finding revealed decreased white matter in the fornix, which has been linked to several key symptom clusters associated with $\mathrm{dPD}$, such as cognitive impairment and apathy (Hollocks et al., 2015).

It is quite striking that significant differences in brain circuitry emerged between individuals with very small (though statistically significant) differences in depressive symptom presentation. The mean Geriatric Depression Scores (GDS) differed by only two points between the "depressed" and "non-depressed" groups. Moreover, the "depressed" group only endorsed mild symptoms, with a mean GDS score of $5.2(\mathrm{SD} \pm 0.04)$ on a 15-point scale (Sheikh and Yesavage, 1986) and were not formally assessed for the presence or absence of a DSM-5 depressive disorder.

Thus, we see that important differences in brain circuitry may emerge even in mild dPD, 
underscoring the clear need for early, consistent, and comprehensive screening of mood symptoms as part of the standard of care for all PWP. Early detection and intervention are needed in order to attenuate the pernicious effects of sub-optimally treated depression on all aspects of PD care. It is only a matter of time until early brain changes observed via imaging translate into the physiological (heart palpitations), cognitive (negative thoughts), behavioral (avoidance), and emotional (chronic feelings of emptiness) symptoms of depression that have major functional impact (Dobkin et al., 2018).

Overall, the findings of Ansari et al. add to the growing literature enhancing understanding of neurocircuitry involved in dPD (Wen et al., 2016). Advanced knowledge regarding the relative contribution of specific neural pathways to dPD may ultimately lead to better therapeutic targets (e.g., self-schemas and emotional processing deficits can be direct targets of psychotherapy) and more precise depression treatment recommendations, particularly for those with atypical symptom presentation, history of treatment resistance, or early cognitive decline. Future studies also have great potential to elucidate underlying neural mechanisms with different implications for depression treatment at various stages of disease progression.

Yet, correlates and risk factors for the psychiatric complications in PD extend well beyond neurobiological factors. The motor symptoms of PD create a progressive set of functional limitations that complicate active, meaningful, and safe engagement in day-to-day life, which can lead to a changed sense of self and altered perspectives on the future. These physical challenges, in conjunction with unique illness-related beliefs and behavioral coping efforts, have significant effects on overall mental health. The studies by Gazibara and colleagues and Vescovelli et al. provide important illustrations of these issues.

\section{Fear of falling: An illustrative example of PD anxiety}

Gazibara and colleagues explored changes in fear of falling (FOF) in PD in a two-year prospective cohort study. In this sample of 120 participants with no history of falls at baseline, FOF significantly increased over two years. The development of recurrent falls in year 1 was highly predictive of increased FOF in year 2, independent of other variables. After two years, recurrent fallers lost confidence in their ability to independently perform the majority of activities of daily living. As FOF is a clear risk factor for future falls (Mak and Pang, 2009; Young and Williams, 2015), frequent fallers may be most vulnerable to detrimental effects of this specific form of anticipatory anxiety (FOF).

Of note, the complex relationship between FOF, depression, and more generalized forms of anxiety was only partially explored. While baseline rates of depression and anxiety were quite low, with Hamilton Depression and Anxiety Scale scores approaching the normal range (HAMD mean of 9 and HAMA mean of 4), and were not predictive of future FOF, changes in these emotional states were not prospectively monitored over time. Depression and anxiety, however, are key constructs to consider in FOF research, as negative mood states significantly increase risk for making negative predictions about the future, including heightened anticipation regarding future falls and low perceptions of self-efficacy for safe participation in activities of daily living (Young and Williams, 2015). Indeed, in our work as clinical psychologists specializing in PD mental health, we find FOF to be intricately related to more generalized forms of anxiety and depression, as well as actual falls, in a vicious cycle. It is not just simply the presence of a physical symptom (in this case, recurrent falls) but the meaning, interpretation, and feared predictions around the symptom that drive mood, quality of life, and functional limitations (Dobkin et al., 2018). Depression and anxiety may also negatively influence attendance at and home practice of Physical and Occupational Therapy (PT/OT), further increasing fall risk (Jayasinghe et al., 2014).

As such, comprehensive multidisciplinary care, inclusive of mental health, may be required to optimize fall prevention. While PT/OT are the gold standard treatments in this domain, negative cognitions related to FOF may block treatment progress, skills practice, and implementation of meaningful behavior changes at home (Jayasinghe et al., 2014). Ongoing collaboration between neurologists, PTs, OTs, and mental health providers may most effectively address physical and emotional risk factors for falls (van der Marck et al., 2014). Falls prevention may further be enhanced by the routine assessment of FOF (as a narrowly defined subtype of PD anxiety), in addition to actual falls, during regularly scheduled PD clinic visits (across all disciplines), especially in frequent fallers, who are at highest risk.

\section{PD mental health: A multifaceted construct}

FOF is one of many issues faced by PWP that challenge their ability to meaningfully engage in daily routines, raising questions about the ultimate impact that these struggles have on sense of 
well-being. While it is well established that PWP experience more emotional distress compared to other geriatric and medical groups (e.g. Tanberg et al.,1996), the focus on a broader concept of psychological well-being has been less of a focus in research. Building upon past crosssectional studies, Vescovelli et al. demonstrated that PWP reported more depression, anxiety, and hostility, as well as decreased life satisfaction and quality of life, compared to age matched controls with non-neurological medical conditions. Yet, surprisingly, PWP also reported higher levels of overall global psychological well-being, compared to their counterparts. This novel and unexpected finding warrants further exploration as it bears significant assessment and treatment implications.

While it is difficult to fully contextualize these results, which appear to stand in clear contrast to one another, two possible explanations come to mind. First, mental health is a multifaceted construct, not an all or nothing phenomenon, and much remains to be learned about barriers and facilitators to optimal mental health in PD. Although emotional distress is quite prevalent in PD, it is also common for people to simultaneously experience different feelings and thoughts. Therefore, it follows that some PWP may be able to maintain a general sense of wellbeing, even in the presence of negative emotions (especially if these negative feelings are fleeting vs. chronic), and do not view themselves as defined by PD. Perhaps, the experience of overcoming unique PD-related challenges leads to enhanced sense of self-efficacy and personal growth for a subset of the PD population, even in the presence of some degree of emotional distress. Second, all participants in the Vescovelli et al. study were essentially "healthy controls" from the psychiatric perspective. The mean scores on depression and anxiety measures were quite low for all participants and individuals diagnosed with a formal depression or anxiety disorder were excluded. Findings about global psychological well-being may have been much different in a PD sub-group with moderate to severe psychiatric concerns.

Though not without limitations, this study underscores several important points. First, depression, anxiety, and physical impairments may complicate, but not preclude, all aspects of healthy psychological well-being in PD. And similar to the studies of Ansari et al. and Gazibara et al. above, this research again highlights the importance of routine and careful assessment of anxiety and depression at each and every routine office visit, even for those who seem to be "doing OK." Last, the variability reflected in this data set underscores that there is no one size fits all approach to mental health treatment in PD. A comprehensive case-by-case assessment must guide appropriate treatment selection for each individual PWP. In addition to targeting core symptoms of depression and anxiety, multidisciplinary interventions can directly focus on many of the broader components of psychological wellbeing, such as environmental mastery, greater purpose and connections with others in daily routines, and adaptive views on oneself, based on individual need.

\section{Conclusions}

In summary, the articles in this issue provide a wide-range lens on the psychiatric aspects of PD, ranging from neurobiological aspects of $\mathrm{dPD}$, to key challenges such as FOF, and to broader views of quality of life and well-being. The picture that emerges is one that clearly depicts the essential consideration of psychiatric aspects of $\mathrm{PD}$ as part of the overall management of the illness. Together, study findings point to key directions in the field. These include research that expands our understanding of psychiatric aspects of PD, with a particular emphasis on identifying biological and psychosocial targets that are modifiable through the use of PD-informed multidisciplinary interventions. Importantly, as the field continues to advance its understanding of psychiatric aspects of PD, it is essential that all multidisciplinary providers develop standardized procedures to improve the assessment and identification of these issues as a key component of routine care.

\section{Roseanne D. Dobkin ${ }^{1}$ and Alejandro Interian ${ }^{1,2}$}

${ }^{I}$ Rutgers, The State University of New Jersey, Robert Wood Johnson Medical School, Piscataway, NJ, USA

${ }^{2}$ VA New Jersey Healthcare System, Mental Health and Behavioral Sciences, Lyons, NJ, USA

\section{References}

Ansari, M., Moradi, S. A., Sherbaf, F. G., Hedayatnia, A. and Aarabi, M. H. (2019). Comparison of structural connectivity in Parkinson's disease with depressive symptoms versus non-depressed: a diffusion MRI connectometry study. International Psychogeriatrics, 31, 5-12.

Cohen, E. V. et al. (2016). Interprofessional education increases knowledge, promotes team building, and changes practice in the care of Parkinson's disease. Parkinsonism $\mathcal{G}$ Related Disorders, 22, 21-27.

Dobkin, R. D., Interian, A., Durland, J. L., Gara, M. A. and Menza, M. A. (2018). Personalized telemedicine for depression in Parkinson's disease: a pilot trial. Fournal of Geriatric Psychiatry and Neurology, 31, 171-176. 
Frodl, T. et al. (2010). Functional connectivity bias of the orbitofrontal cortex in drug-free patients with major depression. Biological Psychiatry, 67, 161-167.

Gazibara, T. et al. (2019). Change in fear of falling in Parkinson's disease: a two-year prospective cohort study. International Psychogeriatrics, 31, 13-20.

Hollocks, M. J. et al. (2015). Differential relationships between apathy and depression with white matter microstructural changes and functional outcomes. Brain, 138, 3803-3815.

Jayasinghe, N. et al. (2014). Exposure-based CBT for older adults after fall injury: description of a manualized, time-limited intervention for anxiety. Cognitive and Behavioral Practice, 21, 432-445.

Mak, M. K. and Pang, M. Y. (2009). Fear of falling is independently associated with recurrent falls in patients with Parkinson's disease: a 1-year prospective study. fournal of Neurology, 256, 1689-1695.

Pontone, G. M. et al. (2016). The longitudinal impact of depression on disability in Parkinson disease. International Fournal of Geriatric Psychiatry, 31, 458-465.

Santos-García, D. and De la Fuente-Fernández, R. (2015). Factors contributing to caregivers' stress and burden in Parkinson's disease. Acta Neurologica Scandinavica, 131, 203-210.

Sauerbier, A. and Chaudhuri, K. R. (2014). Non-motor symptoms: the core of multi-morbid Parkinson's disease. British fournal of Hospital Medicine, 75, $18-24$.

Sheikh, J. I. and Yesavage, J. A. (1986). Geriatric Depression Scale (GDS). Recent evidence and development of a shorter version. In T. L. Brink (ed.), Clinical Gerontology: A Guide to Assessment and Intervention (pp. 165-173). New York: The Haworth Press, Inc.

Tanberg, E., Larsen, J. P., Aarsland, D. and Cummings, J. L. (1996). The occurrence of depression in Parkinson's disease: a community-based study. Archives of Neurology, 54, 175-179.

van der Marck, M. A. et al. (2014). Consensus-based clinical practice recommendations for the examination and management of falls in patients with Parkinson's disease. Parkinsonism E Related Disorders, 20, 360-369.

van Uem, J. M. et al. (2016). Health-related quality of life in patients with Parkinson's disease-a systematic review based on the ICF model. Neuroscience $\mathcal{E}$ Biobehavioral Reviews, 61, 26-34.

Vescovelli, F., Sarti, D. and Ruini, C. (2019). Well-being and distress of patients with Parkinson's disease: a comparative investigation. International Psychogeriatrics, 31, 21-30.

Weintraub, D. and Burn, D. J. (2011). Parkinson's disease: the quintessential neuropsychiatric disorder. Movement Disorders, 26, 1022-1031.

Wen, M. C., Chan, L. L., Tan, L. C. S. and Tan, E. K. (2016). Depression, anxiety, and apathy in Parkinson's disease: insights from neuroimaging studies. European fournal of Neurology, 23, 1001-1019.

Young, W. R. and Williams, A. M. (2015). How fear of falling can increase fall-risk in older adults: applying psychological theory to practical observations. Gait $\mathcal{E}$ Posture, 41, 7-12.

Zis, P., Erro, R., Walton, C. C., Sauerbier, A. and Chaudhuri, K. R. (2015). The range and nature of non-motor symptoms in drug-naive Parkinson's disease patients: a state-of-the-art systematic review. npj Parkinson's Disease, 1, 15013. 